\title{
Coexistent Crohn's disease and sigmoid diverticulosis
}

\author{
Jane McCue' ${ }^{1}$, M.J. Coppen ${ }^{2}$, S.A. Rasbridge ${ }^{2}$ and M.R. Lock ${ }^{1}$ \\ Departments of ${ }^{1}$ Surgery and ${ }^{2}$ Histopathology, Whittington Hospital, London N19, UK.
}

\begin{abstract}
Summary: This study reports six patients with a diagnosis of diverticular disease with associated localized Crohn's colitis who were all treated by segmental resection. Two patients died in the post-operative period from disease unrelated to their colonic pathology. The remaining four patients remain well, show no signs of recurrent disease and have required no further surgery. The behaviour and significance of the two conditions occurring in the same patient is discussed.
\end{abstract}

\section{Introduction}

Crohn's disease of the colon has been diagnosed with increasing frequency in recent years. ${ }^{1-3}$ Diverticular disease is also commonly found in the large intestine, especially of elderly patients, and predictably the two diseases sometimes coexist. Although the difficulty of obtaining a diagnosis between localized Crohn's colitis and diverticular disease is well recognized ${ }^{4-7}$ scant attention has been paid in previous publications to the coexistence of these conditions.

Between 1977 and 1986 six patients with combined diverticulosis and localized Crohn's disease involving the sigmoid colon were diagnosed. This paper describes the clinical presentation, radiological, operative and histological findings and the management of these patients. The significance of dual pathology and suggested guidelines for management are addressed.

\section{Patients and methods}

This retrospective study reviews the case notes and histological specimens of six patients who were seen at the Whittington Hospital between 1977 and 1986 with a diagnosis of combined diverticular disease of the sigmoid colon and localized Crohn's disease.

The patients were submitted to routine follow-up in the outpatient department. The radiological studies were re-examined and the histopathological specimens reviewed by one pathologist (MJC) to verify the diagnosis.

Correspondence: Miss J.L. McCue, M.B., B.S., F.R.C.S., Department of Surgery, Homerton Hospital, Homerton Grove, London E9, UK.

Accepted: 30 March 1989

\section{Results}

\section{Clinical features (Table I)}

There were six patients, four men and two women Their mean age was 66.2 years (50-77 years). The average duration of symptoms prior to diagnosis was 27 weeks ( 1 week to 16 months) plus one patient who was asymptomatic (Patient 4) in whom the diagnosis was made at operation for an incidental abdomina aortic aneurysm.

Pain was the most frequent symptom occurring in four patients. Three patients presented with diarrhoea whilst one complained of constipation; in two patients the bowel habit was unchanged. Three had noticed significant weight loss. One patient presented with recurrent urinary tract infections due to a colovesical fistula (Patient 1).

The signs at presentation were varied. One patient had generalized peritonitis with free intra-peritoneal gas on a plain abdominal X-ray (Patient 5) whilst another had large bowel obstruction (Patient 6). Of the remaining four patients, two had a palpable mass in the left iliac fossa. There were no patients with perianal problems or any extra-intestinal manifestations of Crohn's disease.

Sigmoidoscopy was abnormal in one patient alone (Patient 2), in whom the changes were suggestive of Crohn's colitis. Urgency precluded barium enema in three patients but abnormal barium studies were seen in the remaining three patients. In one case a tight stricture was seen (Patient 3), whilst the other two showed marked diverticular disease with probable additional inflammatory bowel disease in one patient (Patient 2).

Two patients underwent emergency laparotomy. In both instances the operative impression was of divert-

(C) The Fellowship of Postgraduate Medicine, 1989 
Table I Clinical features of combined diverticular disease and Crohn's

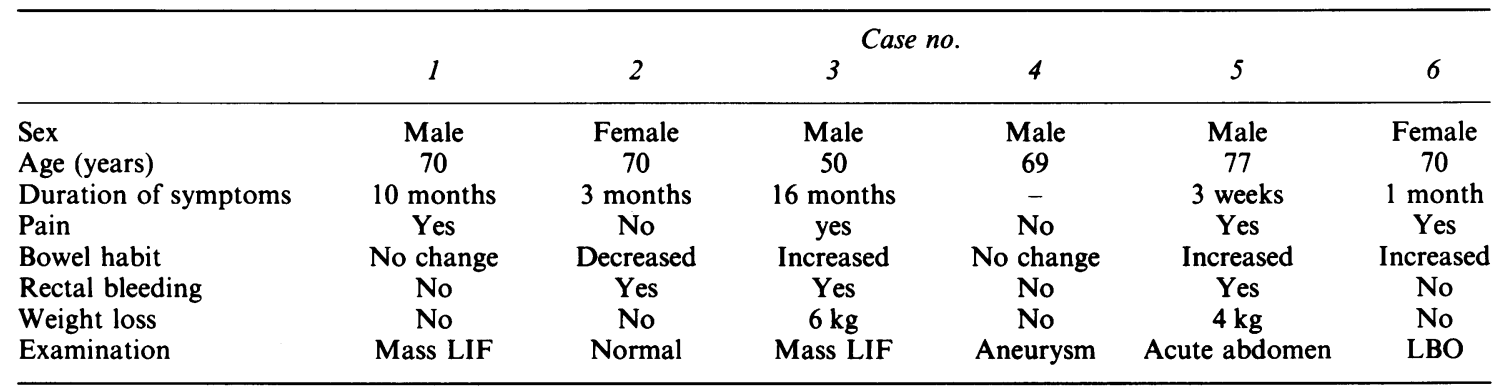

LIF = left iliac fossa; LBO = large bowel obstruction

iculitis complicated in one case by large bowel obstruction above a mass in the sigmoid (Patient 6). In the other patient there was purulent peritonitis secondary to perforation (Patient 5). Both these patients underwent Hartmann's procedure.

In the four patients who underwent elective surgery an operative diagnosis of carcinoma was made in two and diverticulitis in two. In one of the latter cases a colovesical fistula was found (Patient 1). All underwent resection and primary anastomosis. In all patients the disease process was limited to the sigmoid colon.

\section{Histology}

The diagnosis of Crohn's disease and diverticular disease was made by examination of colonic resection specimens from all six patients. In each case thickening of the muscularis propria of the large bowel and diverticula were seen macroscopically. In one case a segment of small intestine with an adherent segment of diseased colon was received (Patient 3); in another a $4 \mathrm{~cm}$ long fistula track was present within the large bowel wall (Patient 1). The most reliable microscopic criteria for the diagnosis of Crohn's disease were made on a combination of histological features, the most important being epitheloid granulomata, transmural inflammation, fissuring and lymphoid aggregates. ${ }^{8,9}$ These were present in all six patients, in conjunction with the features of diverticular disease, i.e. diverticular and hypertrophy of the muscularis propria (see Figures 1 and 2). Furthermore one case showed perforation (Patient 5) while the lymph nodes of three other cases contained granulomata. The rectal biopsy of one patient taken prior to surgery showed features of Crohn's disease (Patient 2).

No evidence of caseation or of foreign body reaction was seen in any of the resection specimens. Granuloma formation alone was not thought diagnostic of Crohn's disease as it has been noted in other colonic conditions including diverticulitis ${ }^{22}$.

\section{Outcome}

There were two deaths unrelated to the Crohn's disease. The patient with large bowel obstruction (Patient 6) died from an upper gastrointestinal haemorrhage shortly after discharge from hospital. The patient who underwent sigmoid resection instead

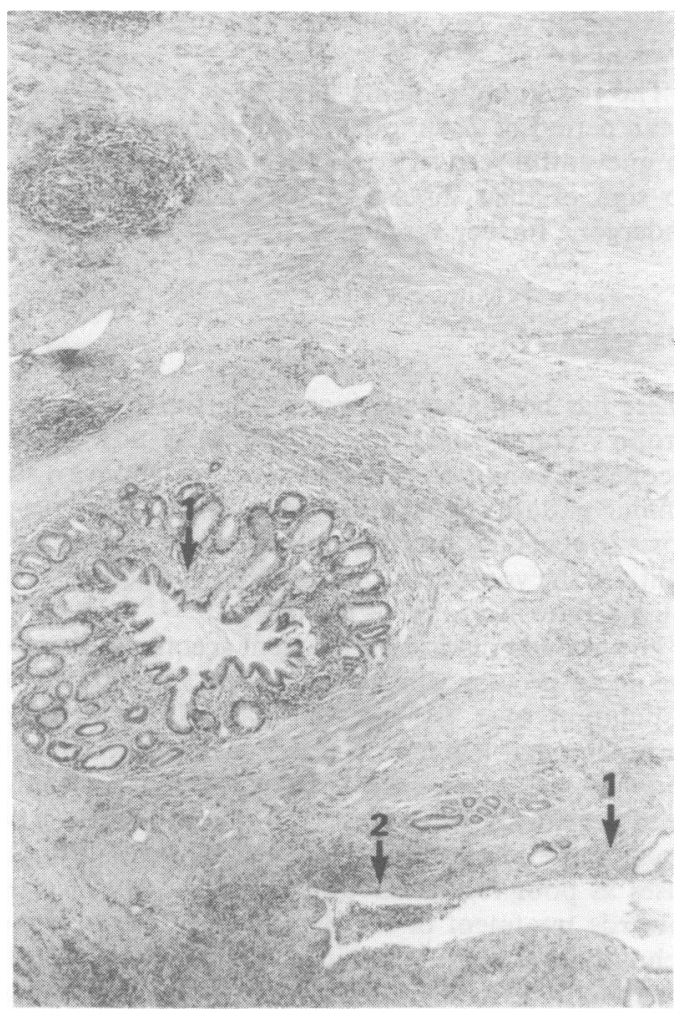

Figure 1 Representative transverse section through colon wall showing lymphoid aggregates, muscle hypertrophy and two diverticula (1), one demonstrating mucosal ulceration (2). Magnification $\times 14$. 


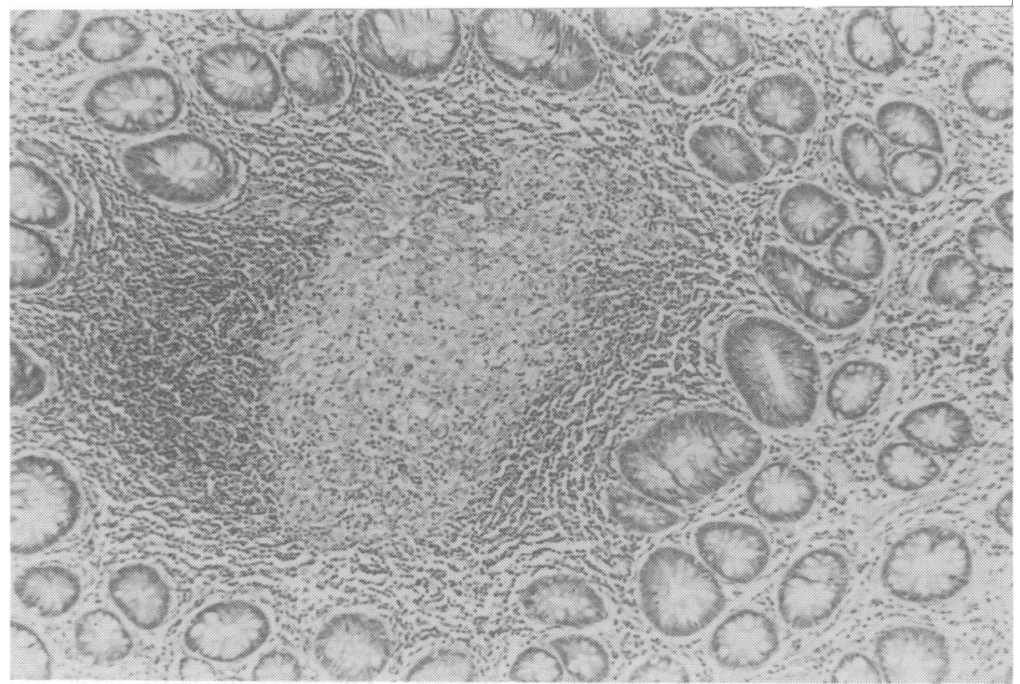

Figure 2 Non-caseating granuloma, typical of Crohn's disease, seen within the mucosa. Magnification $\times 40$.

of intended aortic aneurysm surgery died on the tenth post-operative day when the aneurysm ruptured (Patient 4).

The remaining patients have been followed for a mean period of 22 months (9-42 months). All made an uneventful recovery and have remained well with no sign of their disease or recurrence. None have undergone further surgery.

\section{Discussion}

There has been a steady increase in the incidence of Crohn's colitis since the $1960 \mathrm{~s}^{1,3}$ It is now a well recognized condition in the elderly, ${ }^{7,10}$ in whom a distal colonic pattern is seen more frequently. ${ }^{11,12}$ The prognosis is generally more favourable than for Crohn's colitis in the younger patient and it is considered to be a less aggressive form of the disease. ${ }^{13}$

Diverticular disease is a frequent accompaniment to the aging process, affecting between $35-50 \%$ of the population over the age of $60 .^{14,15}$ It is therefore surprising that less than 70 cases of combined diverticular disease in association with Crohn's disease have been previously recorded., ${ }^{9}, 16,17$ These reports have stressed the importance of correct diagnosis in such cases as poor results have followed surgery directed towards treatment of the diverticular disease alone. Schmidt observed 7 significant post-operative problems out of 13 cases who underwent resection ${ }^{9}$ whilst Berman claimed an $84 \%$ recurrent operative rate following conservative surgery. ${ }^{18}$ The majority of his cases, however, had simply undergone proximal diversion and not resection.
The results of this study are in strong contrast to those series. In the surviving patients there was only one minor post-operative complication (a wound infection). To date there have been no recurrences which is surprising despite the relatively short current follow-up.

These results are in accord with the few reported cases of resection for genuine localized Crohn's disease of the colon without diverticular disease. Goligher reports no recurrences in five cases undergoing resection for isolated left colonic disease. Shapiro similarly observed no recurrences after surgery for seven cases of segmental disease ${ }^{7}$ whilst Sanfey saw only a solitary recurrence after excision of six cases of Crohn's colitis localized to the sigmoid; ${ }^{19}$ an overall recurrence rate of less than $5 \%$.

This study has differed in several aspects of the clinical manifestation of the disease as we have not observed a single case with peri-anal disease or systemic evidence of Crohn's. In previous reports the incidence of peri-anal disease in Crohn's colitis has been as high as $94 \%{ }^{20}$ This suggests that the localized Crohn's disease seen in this study may well be a different clinical variant of Crohn's colitis, which may be inherently less aggressive.

It is widely agreed that colonic Crohn's disease in the elderly carries a good prognosis except when perforation occurs. When Crohn's disease and diverticula occur in the same segment of colon it seems likely to facilitate perforation. In Crohn's disease apthous ulceration or fissures can rapidly breech the mucosa of the diverticulum to produce peridiverticulitis or a para-colic abscess. Meyers has calculated up to a ten-fold increase in the expected rate of diverticulitis in 
such cases. $^{17}$ Free perforation with generalized peritonitis may also occur, as seen at presentation in one of the cases in this article. This is usually a rare event in colonic Crohn's as the full thickness inflammation attracts adjacent structures to the vicinity to localize any infection. An additional factor in perforation may be that stricturing due to diverticular disease may lead to increased intraluminal pressure which may rupture a fissure/ulcer. The treatment of choice appears to be resection and anastomosis where possible. This has a mortality of only $3.7 \%$ compared with $39 \%$ resulting from simple suture. ${ }^{21}$

\section{References}

1. Goligher, J.C. Crohn's disease of the large intestine. Adv Surg 1977, 2: 87-99.

2. Harries, A.D., Baird, A., Rhodes, J. \& Mayberry, J.F. Has the rising incidence of Crohn's disease reached a plateau? Br Med J 1982, 284: 235.

3. Mee, A.S. Crohn's disease of the colon. Problems and delays in diagnosis. SA Med J 1982, 61: 746-750.

4. Harper, P.C., McAuliffe, T.L. \& Beeken, W.L. Crohn's disease in the elderly. A statistical comparison with younger patients matched for sex and duration of disease. Arch Int Med 1986, 146: 753-755.

5. Hoffman, W.A. \& Rosenberg, M.A. Granulomatous colitis in the elderly. Am J Gastroenterol 1972, 59: 508-518.

6. Hutton, I. Crohn's disease in the elderly. Proc R Soc Med 1975, 68: 329-330.

7. Shapiro, P.A., Peppercorn, M.A., Antonioli, D.A., Joffe, N. \& Goldman, H. Crohn's disease in the elderly. Am J Gastroenterol 1981, 76: 132-137.

8. Lockhart-Mummery, H.E. \& Morson, B.C. Crohn's disease (regional enteritis) of the large intestine and its distinction from ulcerative colitis. Gut 1960, 1: 87-105.

9. Schmidt, G.T., Lennard-Jones, J.E., Morson, B.C. \& Young, A.C. Crohn's disease of the colon and its distinction from diverticulitis. Gut 1968, 9: 7-16.

10. Tchircow, G., Lavery, I.C. \& Fazio, V.W. Crohn's disease in the elderly. Dis Colon Rectum 1983, 26: $177-181$.

11. Carr, N. \& Schofield, P.F. Inflammatory bowel disease in the elderly patient. Br J Surg 1982, 69: 2235.

12. Fabricius, P.J., Gyde, S.N., Keighley, M.R.B. et al. Crohn's disease in the elderly. Gut 1985, 26: 461-465.
In conclusion this study involved six cases of localized colonic Crohn's disease in association with diverticulosis. The findings suggest the concept that whilst localized Crohn's colitis in the elderly is less aggressive and shows none of the systemic manifestations of Crohn's disease, nevertheless an increasing rate of perforation occurs when the disease is found in conjunction with sigmoid diverticula.

The results of the study strongly suggest that, even in cases of perforation, localized resection is the treatment of choice for colonic diverticular disease in association with localized Crohn's colitis.

13. Rhodes, J. \& Rose, J. Crohn's disease in the elderly. Br Med J 1985, 291: 1149.

14. Manousos, O.N., Truelove, S.C. \& Lumsden, K. Prevalence of colonic diverticulosis in general population of Oxford area. Br Med J 1967, 3: 336-344.

15. Hughes, L.E. Postmortem survey of diverticular disease of the colon. 1 Diverticulosis and diverticulitis. Gut 1969 , 10: $336-344$.

16. Marshak, R.H., Janowitz, H.D. \& Present, D.H. Granulomatous colitis in association with diverticula. $N$ Engl J Med 1970, 283: 1080-1083.

17. Meyers, M.A., Alonso, D.R., Morson, B.C. \& Bartram, C. Pathogenesis of diverticulitis complicating granulomatous colitis. Gastroenterology 1978, 74: 24-31.

18. Berman, I.R., Corman, M.L., Coller, J.A. \& Veidenheimer, M.C. Late onset Crohn's disease in patients with colonic diverticulitis. Dis Colon Rectum 1979, 22: 524-529.

19. Sanfey, F., Bayless, T.M. \& Cameron, J.L. Crohn's disease of the colon. Is there a role for limited resection? Am J Surg 1984, 147: 38-42.

20. Buchman, P. \& Alexander Williams, J. Classification of perianal Crohn's disease. Clin Gastroenterol 1980, 9: 323-330.

21. Greenstein, A.J., Mann, D., Sachar, D.B. \& Aufses, A.H. Free perforation in Crohn's disease: 1 . A survey of 99 cases. Am J Gastroenterol 1985, 80: 682-689.

22. Morgenstern, L., Weiner, R. \& Michel, S.L. Malignant diverticulitis: a clinical entity. Arch Surg 1979, 114: 1112-1116. 